\title{
Behavioral and psychological symptoms and cognitive decline in patients with amnestic MCI and mild AD: a two-year follow-up study
}

\author{
Cornelia Pocnet, Jean-Philippe Antonietti, ${ }^{1}$ Alessia Donati, ${ }^{2}$ Julius Popp, ${ }^{2}$ \\ Jérôme Rossier ${ }^{1}$ and Armin von Gunten ${ }^{2}$ \\ ${ }^{1}$ Institute of Psychology, University of Lausanne, Lausanne, Switzerland \\ ${ }^{2}$ Service of Old Age Psychiatry, Department of Psychiatry, Lausanne University Hospital, Lausanne, Switzerland
}

Background: Mild cognitive impairment (MCI) has been defined as a transitional state between normal aging and dementia. In many cases, MCI represents an early stage of developing cognitive impairment. Patients diagnosed with MCI do not meet the criteria for dementia as their general intellect and everyday activities are preserved, although minor changes in instrumental activities of daily living (ADL) may occur. However, they may exhibit significant behavioral and psychological signs and symptoms (BPS), also frequently observed in patients with Alzheimer's disease (AD). Hence, we wondered to what extent specific BPS are associated with cognitive decline in participants with MCI or AD.

Methods: Our sample consisted of 164 participants, including 46 patients with amnestic (single or multidomain) $\mathrm{MCI}$ and 54 patients with $\mathrm{AD}$, as well as 64 control participants without cognitive disorders. Global cognitive performance, BPS, and ADL were assessed using validated clinical methods at baseline and at two-year follow-up.

Results: The BPS variability over the follow-up period was more pronounced in the MCI group than in patients with AD: some BPS improve, others occur newly or worsen, while others still remain unchanged. Moreover, specific changes in BPS were associated with a rapid deterioration of the global cognitive level in MCI patients. In particular, an increase of euphoria, eating disorders, and aberrant motor behavior, as well as worsened sleep quality, predicted a decline in cognitive functioning.

Conclusions: Our findings confirm a higher variability of BPS over time in the MCI group than in AD patients. Moreover, our results provide evidence of associations between specific BPS and cognitive decline in the MCI group that might suggest a risk of conversion of individuals with amnestic MCI to AD.

Key words: behavioral and psychological symptoms, cognitive level, daily living functioning

\section{Introduction}

Often, amnestic single or amnestic multiple domain MCI corresponds to prodromal AD. In patients with MCI, cognitive impairment does not meet the criteria for dementia as their general intellectual functioning and daily life activities remain intact for some time (Winblad et al., 2004).

In studies examining the course of amnestic MCI, the rates of conversion to dementia vary depending on the follow-up period and conceptual definition: $12 \%$ per year for 4 years (Petersen, 2004)

\footnotetext{
Correspondence should be addressed to: Cornelia Pocnet, Institute of Psychology, University of Lausanne, Géopolis, 1015 Lausanne, Switzerland. Email: Cornelia.Pocnet@unil.ch. Received 3 Jun 2014; revision requested 2 Aug 2014; revised version received 9 Dec 2014; accepted 15 Dec 2014. First published online 16 January 2015.
}

and $23 \%$ across two years (Busse et al., 2003). This rate remains comparable with other studies that found that only one-third of individuals with amnestic MCI have an increased risk of AD (Bozoki et al., 2001), whereas others improve, reverting to a normal level of cognitive functioning over a two-year period (Ganguli et al., 2004). Other investigators showed that $34 \%-100 \%$ of individuals with MCI develop AD over 4 to 5 years (Bennett et al., 2002) while across 9.5 years the conversion is $100 \%$ (Morris et al., 2001).

Different criteria have been used to define the features that may predict progression to dementia. In particular, researchers have focused on decline in episodic memory because it is known to be affected earliest and most profoundly in $\mathrm{AD}$ (Bozoki et al., 2001). Moreover, neuroimaging studies of 
Table 1. Differences between the three groups according to level of education and gender variables

\begin{tabular}{|c|c|c|c|c|}
\hline & CONTROLS $(n, \%)$ & $\operatorname{MCI}(n, \%)$ & $\mathrm{AD}(n, \%)$ & $\chi^{2}$ \\
\hline Level of education & & & & $\chi^{2}(4)=51.34, p<0.001$ \\
\hline Compulsory school & $4(7.4 \%)$ & $5(10.9 \%)$ & $30(55.6 \%)$ & \\
\hline Secondary school & $35(54.7 \%)$ & $31(67.4 \%)$ & $20(37 \%)$ & \\
\hline University degree & $25(39.1 \%)$ & $10(21.7 \%)$ & $4(7.4 \%)$ & \\
\hline Gender & & & & $\chi^{2}(2)=3.90, p=0.137$ \\
\hline Women & $35(54.68 \%)$ & $30(65.22 \%)$ & $39(72.22 \%)$ & \\
\hline Men & $29(45.32 \%)$ & $16(34.78 \%)$ & $15(7.78 \%)$ & \\
\hline
\end{tabular}

Note. $n=$ number of participants; $\%=$ the differences between the three groups as a percentage.

MCI individuals suggest that abnormalities in the temporal, parietal, and posterior cingulate regions are most predictive of progression to dementia (Anchisi et al., 2005).

A number of factors may influence the progression from MCI to AD (e.g. Monastero et al., 2007; Ritchie et al., 2010). BPS may be among them. They include depression, anxiety, agitation, apathy, irritability, aberrant motor behavior, or eating disorders, among others. BPS prevalence ranges from $43 \%$ to $59 \%$ in people with MCI (Barnes et al., 2006) and affects $50 \%-80 \%$ of persons with dementia (Lyketsos et al., 2002).

Among the different BPS, apathy seems to be increasingly common as dementia worsens (Teng et al., 2007; Pocnet et al., 2013). The role of depressive symptoms in the progression of MCI to $\mathrm{AD}$ is controversial (Holtzer et al., 2003; Modrego and Ferrandez, 2004; Rozzini et al., 2005; Nguyen and Zonderman, 2006), but higher levels of depressive symptoms were associated with an increased incidence rate of MCI (Barnes et al., 2006; Geda et al., 2006; Monastero et al., 2009) as well as more rapid cognitive decline (Mega et al., 1996; Yaffe et al., 1999). Each additional anxiety symptom almost doubled the risk of conversion from MCI to AD (Palmer et al., 2007). However, other studies have found almost no correlation between BPS and the level of cognitive impairment (Rozzini et al., 2005; Lam et al., 2006).

Thus, the role of specific BPS on cognitive changes in patients with early cognitive impairment remains unclear. Hence, we wished to evaluate the possible influence of changes in specific BPS, in particular depression and apathy, on further cognitive decline in MCI and $\mathrm{AD}$ patients as compared to cognitively healthy individuals.

\section{Methods}

\section{Participants}

The total sample consisted of 164 participants including 46 patients with MCI (amnestic single and multiple MCI) (30 women and 16 men), 54 patients at an early stage of $\mathrm{AD}$ dementia (39 women and $15 \mathrm{men}$ ), and 64 control participants without cognitive disorders (35 women and 29 men). There was no significant difference between the groups according to gender $\left(\chi^{2}(2)=3.97, p=\right.$ $0.137)$. However, the level of education differed in the three groups $\left(\chi^{2}(4)=51.34, p<0.001\right)$ (Table 1).

\section{Materials and procedure}

ASSESSMENT OF BEHAVIORAL AND

\section{PSYCHOLOGICAL SYMPTOMS}

The Neuropsychiatric Inventory (NPI-Q) (Cummings, 1997) was used to define the presence and severity of BPS. The NPI-Q has wide acceptance as a measure of BPS associated with cognitive disorders and good reliability and construct validity (Cummings and McPherson, 2001). It rates symptoms in 12 domains: delusions, hallucinations, agitation, depression, anxiety, euphoria, apathy, disinhibition, irritability, aberrant motor behavior, sleep disturbance, and eating disorders. Each BPS is assessed according to a three-level severity scale ranging from 1 to 3 , yielding a maximal total NPI score of 36 . The NPI is a fully structured informantbased interview used at baseline and at two-year follow-up.

\section{ASSESSMENT OF COGNITIVE STATUS AND}

\section{DAILY LIVING FUNCTIONING}

Initial and follow-up cognitive functioning was evaluated using the Mini-Mental State Examination (MMSE; Folstein et al., 1975) and the Informant Questionnaire on Cognitive Decline (IQCODE; Jorm and Jacomb, 1989). The MMSE permits a quick screening of the patient's global cognitive functioning level. The IQCODE evaluates a subject's cognitive and functional level change. It is a 16-item scale filled out by a relative. Lower MMSE scores and higher IQCODE scores indicate cognitive dysfunction.

Daily living was evaluated by family members twice, two years apart, using the ADL scale (Katz, 
1998) and the Instrumental Activities of Daily Living scale (IADL) for which good reliability and construct validity have been reported (Lawton and Brody, 1969). The ADL is a hierarchical scale including six activities: bathing, dressing, going to the toilet, transferring, continence, and feeding. The IADL is an eight-item scale evaluating the patient's dependency level for activities including shopping, using the public transportation system, cooking, house cleaning, doing laundry, using the phone, taking medication, or managing a budget. Low ADL and IADL scores indicate reduced or lost autonomy.

\section{Procedure}

The individuals with MCI and AD were recruited within the geriatric and gerontopsychiatric structures of the university hospitals of Lausanne and Geneva. The control participants were recruited through newspaper announcements and word of mouth. All the patients had a comprehensive medical, psychiatric, neuropsychological, and psychosocial evaluation. Most often, the patients also had a cerebral magnetic resonance imaging as well as a number of standard laboratory tests. This investigation yielded $\mathrm{AD}$ diagnosis according to the International Classification of Diseases, tenth edition (Word Health Organization, 1993) as well as the National Institute of Neurological and Communicative Disorders and Stroke and the Alzheimer's Disease and Related Disorders Association (NINCDS-ADRDS) criteria (McKhann, 1984). Only patients with a Clinical Dementia Rating scale (CDR) score of 0.5 for MCI and 1 for AD were included (Morris, 1993). Regarding participants with MCI, only those with amnestic single and multiple domain forms were taken into account. The diagnosis of MCI was clinical and based on the criteria proposed by Winblad et al., 2004. MCI was diagnosed in individuals with mild cognitive decline as corroborated by an informant and confirmed by a neuropsychological examination including the MMSE (Folstein et al., 1975), the spontaneous and cued recall 48-items task (Buschke, 1984) to test episodic memory, while to assess executive functions, we used three tests proposed by the GREFEX manual (Godefroy et al., 2010): a verbal fluency task (categorical and literal fluency in 2 minutes), a flexibility task (the Trail Making Test), and an inhibition task (the Stroop test). Only individuals who did not satisfy the NINCDSADRDA criteria for AD or other types of dementia were selected for MCI diagnosis (McKhann et al., 1984). Only patients accompanied by their family members (adult child or spouse caregivers) and older than 55 years were included. Physical illness and major psychiatric disorders that could put the patient at risk such as psychotic illness, bipolar disorder or alcohol dependence were exclusion criteria. Furthermore, we wished to exclude those with elevated anxiety and depression scores as defined by a score equal to or higher than ten on either of the two sub-scales of the Hospital Anxiety and Depression scale (HAD, Zigmond and Snaith, 1983) to rule out as much as possible any major concomitant affective comorbidity deserving a separate affective diagnosis. In addition, school education of less than five years and consent refused by the patient or by their relative are also part of exclusion criteria.

The goals of the project were explained to the participants and their proxies. Among the patients asked to participate in the study, about half agreed to participate and signed the informed consent form, meaning that only people with a basic interest did participate. Proxies had to be family to live in the same household or nearby, to know the participant and their habits well, and to take care of their needs. In the case of mild $\mathrm{AD}$ patients, only the spouse or an adult child taking care of or being in contact with the participant on a daily basis were included. Other family members or professional carers may have intervened occasionally, but on a clearly minor level. Regarding MCI participants, they are still autonomous for daily activities, and the family members participating in this study declared that they are well aware of the habits of their relative. Therefore, the caregivers included were likely to be the pivotal caregivers.

Each participant completed the MMSE twice, two years apart. Their family members completed the NPIQ and the IQCODE, ADL, and IADL scales. Therefore, BPS, cognitive status, and daily living functioning in both the patients and the control participants were assessed identically.

This prospective pilot study was approved by the Ethics Committee of the Faculty of Medicine of the University of Lausanne (Protocol 85/2008). The research complies with the ethical rules of the Swiss Society for Psychology (SSP).

\section{Statistical analysis}

All analyses were performed with SPSS, Version 22, and R ( R Development Core Team, 2009). Descriptive statistics were used to evaluate demographic and clinical characteristics. Then, Spearman correlations between BPS, cognitive level, daily living, and demographic variables (education, gender, and age) were calculated at the beginning (T1) and two years later (T2), and between $\mathrm{T} 1$ and $\mathrm{T} 2$. 
In order to determine BPS change for each group between $\mathrm{T} 1$ and $\mathrm{T} 2$, NPI-Q variables were dichotomized as being absent (value 0) or present (value 1). The differences were then calculated between the measures at $\mathrm{T} 2$ and $\mathrm{T} 1$ (delta) for each group. Delta " -1 " indicates deterioration, " 0 " absence of change and " +1 " improvement. The Wilcoxon test was used to determine possible differences between the three groups of participants for each specific BPS. Differences regarding the changes in the total BPS scores between the three groups were determined using the Kruskal-Wallis non-parametric test and non-parametric multiple comparisons. In addition, we have adjusted for age, gender, and level of education using Poisson regressions to confirm possible differences.

To identify changes in cognitive level (MMSE, IQCODE) and daily living functioning (ADL, and IADL scales) between T2 and T1, the index change (delta) was computed for each group.

We then tried to explain the evolution of the cognitive functioning in amnestic MCI group, considering the large variability of BPS over two years in this group. For this, we used the original non-dichotomized NPI-Q variables to perform multiple regressions. The selection of the most parsimonious model was made using the step-bystep method and the AIC (Akaike Information Criterion) to assess the trade-off between the goodness of fit of the model and the complexity of the model (Akaike, 1974).

\section{Results}

\section{Clinical variables of the three groups at $\mathrm{T} 1$ and $\mathbf{T} 2$}

Table 2 shows the comparisons between the three groups for both the initial and follow-up assessments. For the MCI group, the total scores for ADL, IADL, and MMSE are similar at baseline and at two-year follow-up. This contrasts with the cognitive functional level perceived by their proxies that tends to worsen and with global BPS severity, which almost doubles after two years. AD patients declined for almost all clinical variables. No changes were identified in the control group between the two assessments.

The Spearman and point-biserial correlations between age, gender, education, and total scores of BPS, cognitive level, and daily functioning are shown in Table 3. Gender had no impact on BPS, cognitive level or daily living functioning. However, several correlations between clinical outcomes obtained at either T1 or T2 and between the two assessments were associated with a high $(r \geq 0.50)$ or medium effect size $(r \geq 0.30)$. For

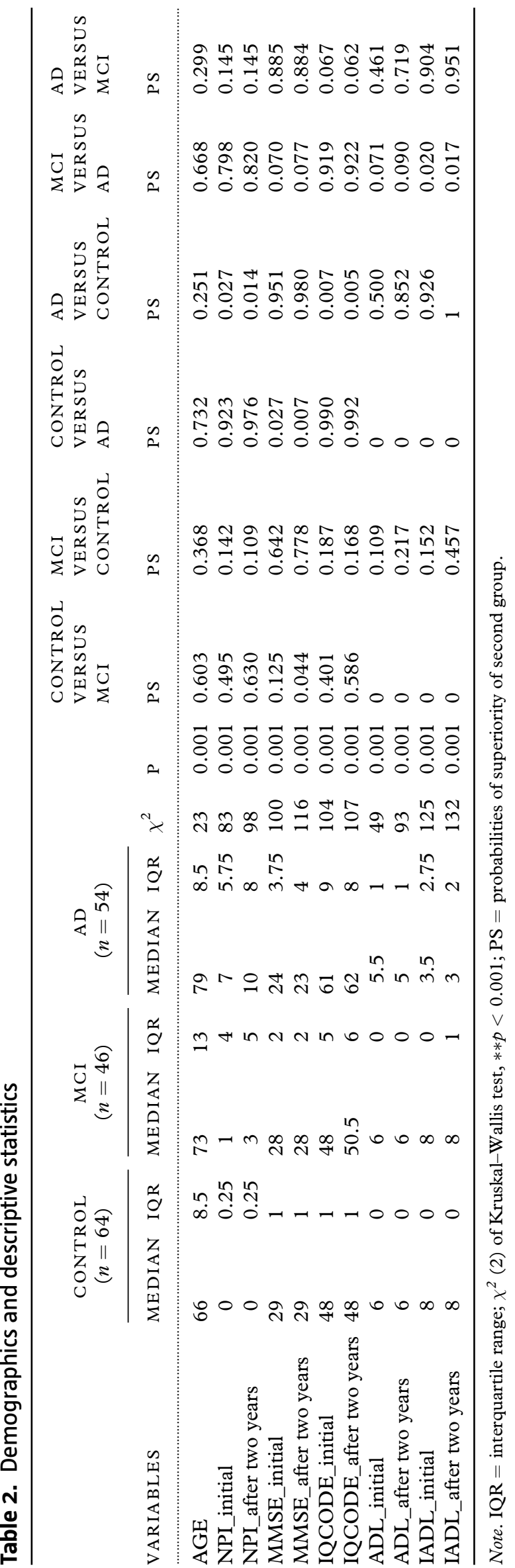




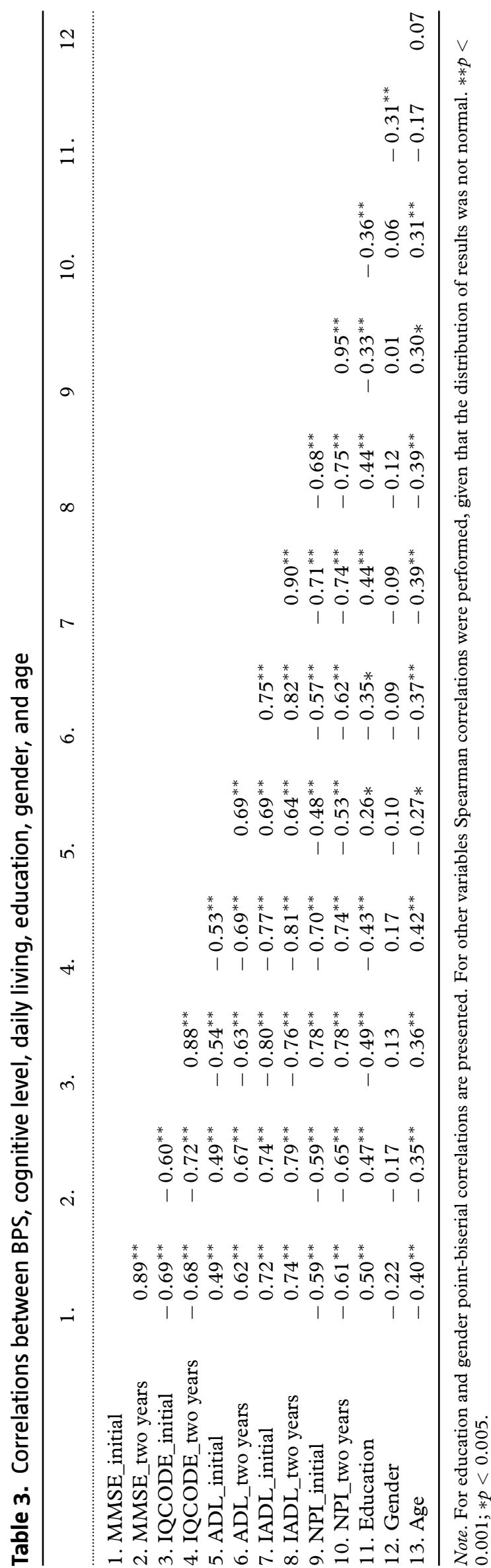

example, the total NPI-Q and IQCODE scores correlated positively with each other, but negatively with the total scores of the MMSE, ADL, and IADL measured both initially, two years later, and between $\mathrm{T} 1$ and $\mathrm{T} 2$ (see Table 3).

\section{Evolution of BPS, cognitive, and daily living functioning in the three groups over two years}

Figure 1 shows significant differences between the three groups regarding the evolution of BPS over two years $\left(\chi^{2}(2)=63.201, p<0.001\right)$, between the control and MCI groups $(z=5.806, p<0.001)$, between MCI and AD patients $(z=5.031, p<$ 0.001 ), but no significant differences between the control and AD groups $(z=0.609, p=0.542)$. Using Poisson regressions and having controlled for age, gender, and level of education, we found that the BPS differences were due only to the variable "group" $\left(\chi^{2}(2)=196.78, p<0.001\right)$ showing that, in the MCI patients, BPS assessed at T2 were clearly distinct from those at $\mathrm{T} 1(\beta=2.863$, $p<0.001)$ as compared to the $\mathrm{AD}$ and control groups. Table 4 indicates that some symptoms newly occurred or worsened in the MCI group (i.e. hallucinations, depression, apathy, irritability, aberrant motor behavior, sleep disturbance, eating disorders, and total scores of symptoms). Some symptoms tended to improve slightly or to remain stable during the two years (i.e. delusions, euphoria, agitation, anxiety, disinhibition).

The MMSE scores of most MCI patients remained almost unchanged over the two years as opposed to the perception of some family members (see Figure 2). However, in the AD patients BPS hardly changed, while the cognitive capacities worsened continuously. All variables remained stable in the control group (see Figures 1 and 2).

\section{Relationship between specific BPS and cognitive or daily living functioning in MCI patients}

Multiple regressions showed that changes in four specific BPS over a two-year period could partly explain the changes of the MMSE score in the MCI group $\left(R^{2}=0.33, F(4,41)=5.09, p=\right.$ 0.001): sleep disturbance $(t(41)=2.32, \beta=$ $2.221, p>0.002)$, euphoria $(t(41)=-2.32, \beta$ $=-2.221, p>0.006)$, eating disorders $(t(41)=$ $-2.54, \beta=-2.570, p>0.01)$, and aberrant motor behavior $(t(41)=-1.89, \beta=-1.41, p>$ $0.06)$. Thus, an increase of euphoria scores, eating disorders, and aberrant motor behavior, as well as worsened sleep quality are related to the progression of cognitive deterioration in patients with MCI (Figure 3). IQCODE, ADL, and IADL scores were not associated with BPS. 


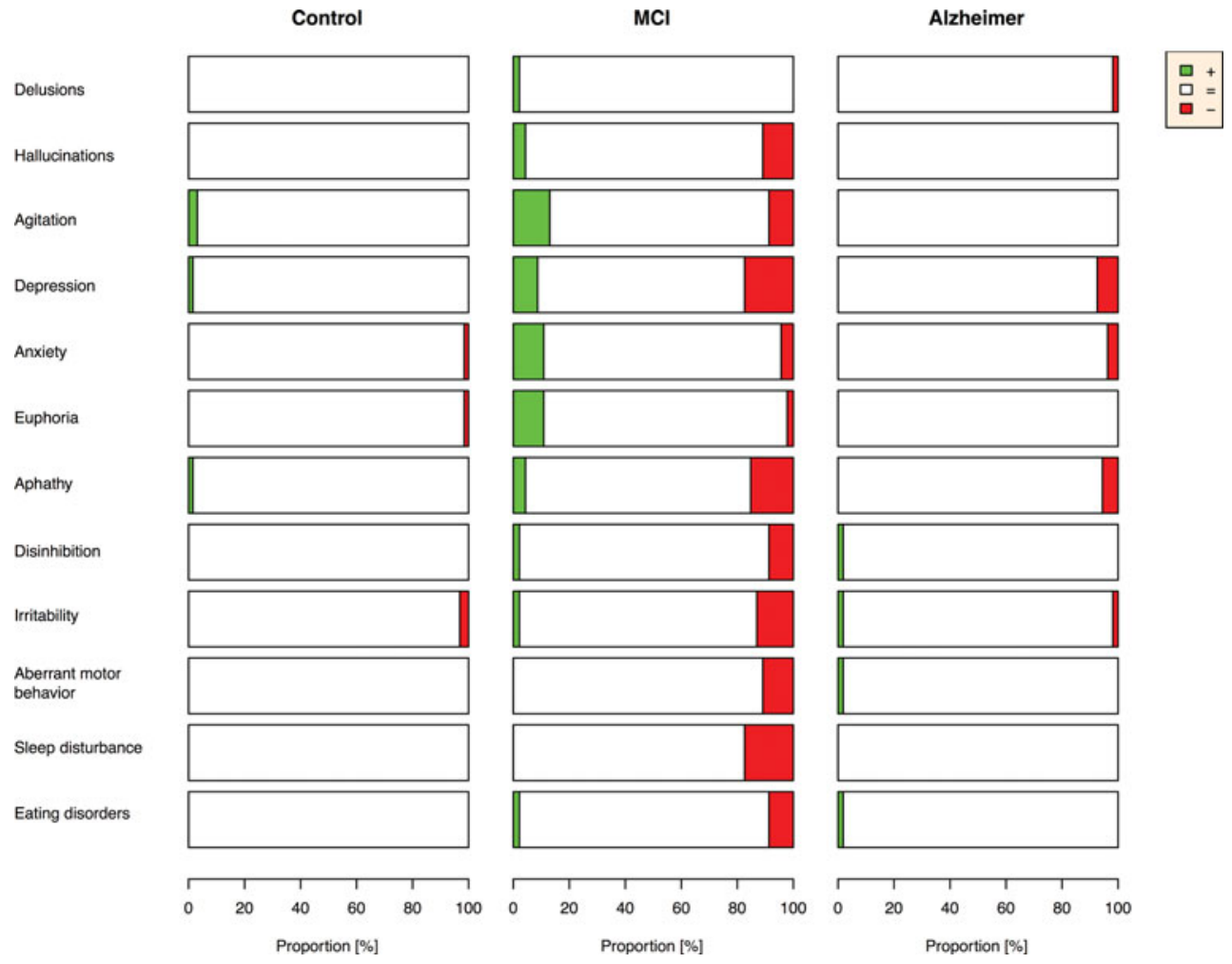

Figure 1. (Colour online) Differences regarding the evolution of BPS over a two-year period in three groups. Note: The colors indicate the direction of the evolution of the symptoms: green or the sign " + " indicate an improvement of scores; red or " -" means worsening; white or " =" means that no change has occurred. The values representing the percentage changes (positive or negative) are indicated in Table 4.

Table 4. Evolution of BPS in participants with amnestic $\mathrm{MCl}$ over two years

\begin{tabular}{|c|c|c|c|c|c|c|c|c|}
\hline $\begin{array}{l}\text { NPI-Q (INITIAL AND } \\
2 \text { YEARS LATER) }\end{array}$ & $\begin{array}{l}\text { MEDIAN } \\
\text { INITIAL }\end{array}$ & $\begin{array}{l}\text { IQR } \\
\text { INITIAL }\end{array}$ & $\begin{array}{l}\text { MEDIAN } \\
\text { AFTER TWO } \\
\text { YEARS }\end{array}$ & $\begin{array}{l}\text { IQR AFTER } \\
\text { TWO YEARS }\end{array}$ & $U$ & $p$ & $\%(-)$ & $\%(+)$ \\
\hline Delusions & 0 & 0 & 0 & 0 & 1 & 0.10 & 0.022 & 0 \\
\hline Hallucinations & 0 & 0 & 0 & 0 & 8 & 0.08 & 0.043 & 0.152 \\
\hline Agitation & 0 & 0 & 0 & 0 & 39 & 0.23 & 0.130 & 0.087 \\
\hline Depression & 0 & 0 & 0 & 1 & 29 & 0.13 & 0.087 & 0.217 \\
\hline Anxiety & 0 & 1 & 0 & 1 & 35 & 0.43 & 0.130 & 0.087 \\
\hline Euphoria & 0 & 0 & 0 & 0 & 18 & 0.12 & 0.109 & 0.022 \\
\hline Apathy & 0 & 1 & 1 & 2 & 21 & 0.01 & 0.043 & 0.304 \\
\hline Disinhibition & 0 & 0 & 0 & 0 & 2 & 0.17 & 0.022 & 0.087 \\
\hline Irritability & 0 & 0 & 0 & 1 & 20 & 0.12 & 0.065 & 0.196 \\
\hline Aberrant motor behavior & 0 & 0 & 0 & 0 & 3 & 0.05 & 0 & 0.109 \\
\hline Sleep disturbance & 0 & 0 & 0 & 0.75 & 3 & 0.01 & 0.022 & 0.196 \\
\hline Eating disorders & 0 & 0 & 0 & 0 & 2 & 0.16 & 0.022 & 0.087 \\
\hline NPI-Q total scores & 1 & 4 & 3 & 5 & 30 & 0.001 & 0.087 & 0.522 \\
\hline
\end{tabular}

Note. IQR $=$ interquartile range; $U=$ Wilcoxon Test, $* * * p<0.001 ; * * p<0.01 ; * p<0.05 ; \%(-)=$ the proportion of negative differences means improvement in symptoms; $\%(+)=$ the proportion of positive differences translate into worsening symptoms; $d f=46$.

\section{Discussion}

This study confirms that BPS are frequent in patients with MCI and $\mathrm{AD}$, but that their severity and evolution over two years vary considerably. Although, there are strong correlations between the
$\mathrm{T} 1$ and $\mathrm{T} 2$ measurements, the variability between $\mathrm{T} 1$ and $\mathrm{T} 2$ was more pronounced in those with MCI than in participants with AD. Some BPS tend to improve (delusion and euphoria), others newly occur or worsen (hallucinations, depression, apathy, aberrant motor behavior, sleep disturbance, 

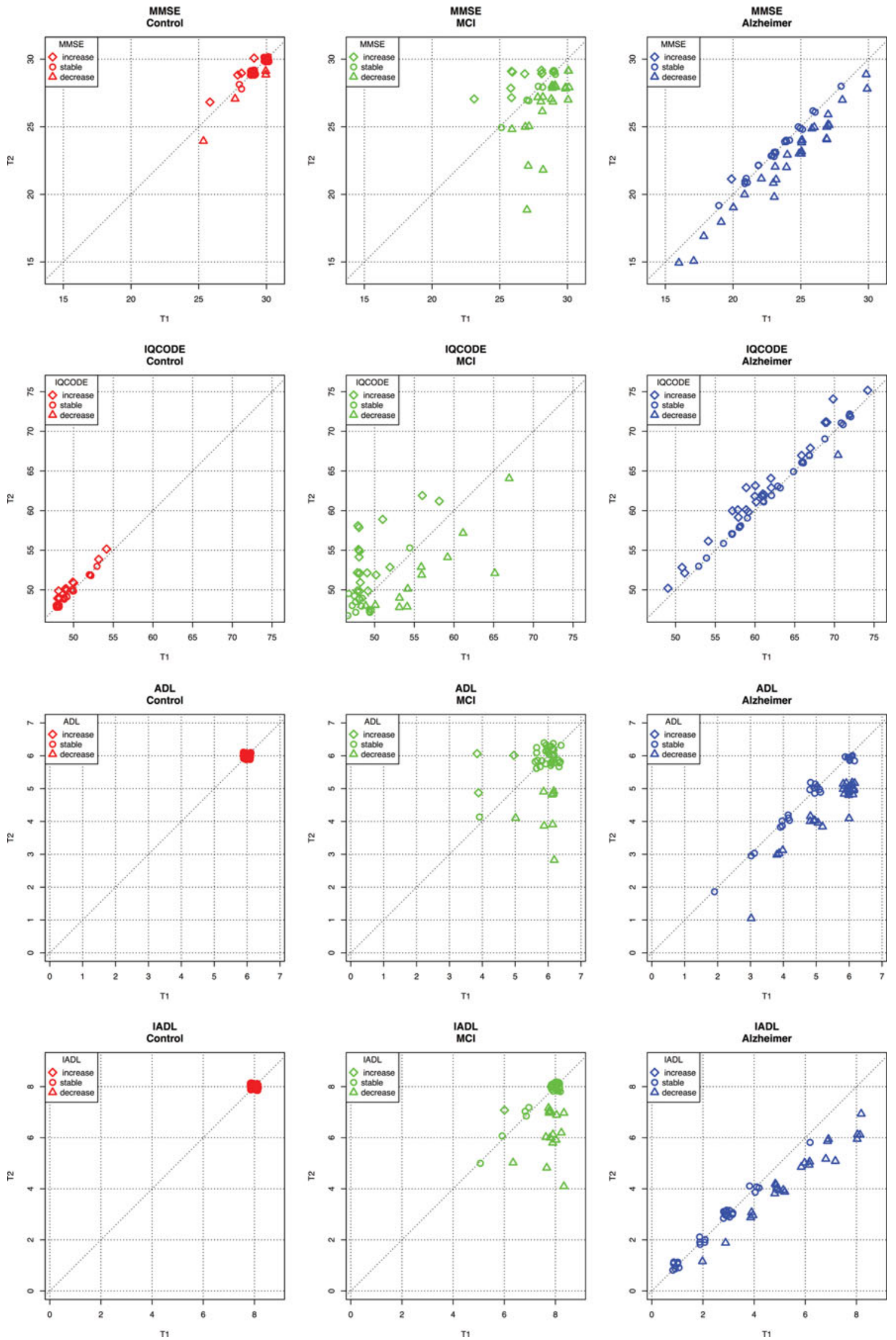

Figure 2. (Colour online) Differences in global cognitive levels and in daily living functioning in the three groups over two years. 

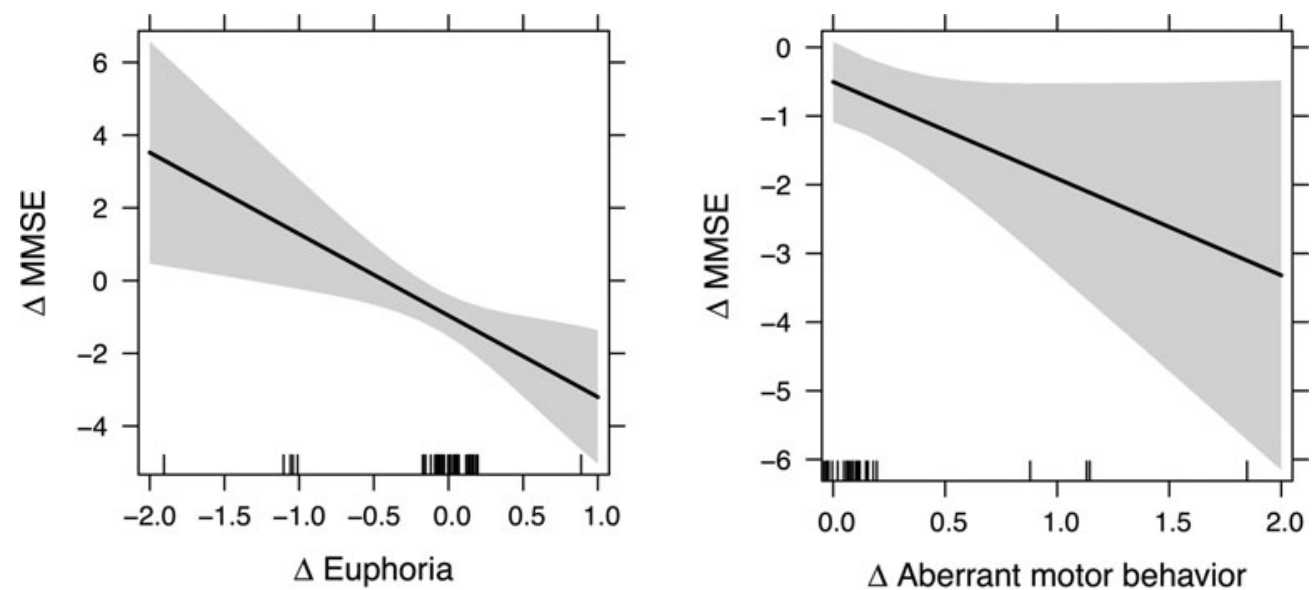

$\Delta$ Aberrant motor behavior

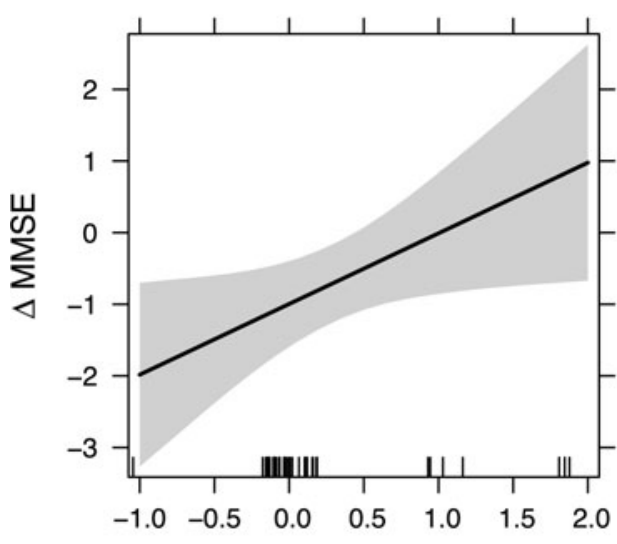

$\Delta$ Sleep disturbance

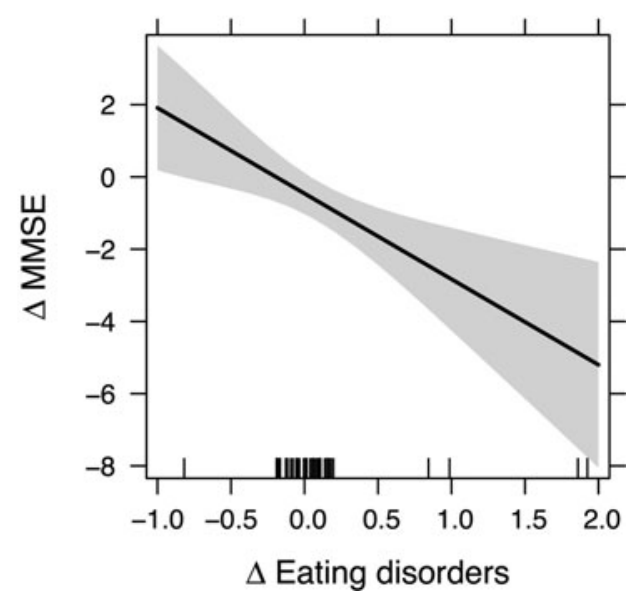

Figure 3. Graph of the influence of specific BPS (NPI-Q) on cognitive status (MMSE) in the MCl group, over a two-year period. Note: The vertical axis represents the change of the MMSE score over two years (delta) and the horizontal axis shows the change of specific BPS (delta), i.e. euphoria, eating disorders, aberrant motor behavior, and sleep disturbance. The straight line represents the interaction between the evolution of these variables (cognitive level and BPS). The symptom changes for each participant with $\mathrm{MCl}$ are shown at the bottom of each figure by a small vertical stroke; the majority of participants with $\mathrm{MCl}$ are concentrated around the 0 (see Figure 2).

irritability, eating disorders, and total scores of symptoms), while others still remain almost unchanged (agitation, anxiety, and disinhibition). Similarly, previous studies showed a non-uniform evolution of specific symptoms in MCI patients (Bozoki et al., 2001; Lyketsos et al., 2002; Petersen, 2004; Palmer et al., 2007; Teng et al., 2007). A partial explanation of this variability could be the pharmacological or non-pharmacological treatments of BPS but, unfortunately, in our study this information is not available.

The current findings raise the question of whether such symptoms reflect specific neuropathological changes in the brain or indicate a subjective reaction to cognitive change. Our findings suggest that some mood symptoms (i.e. anxiety, agitation) that begin very early may represent a reaction to the initial phases of cognitive deterioration. However, others BPS (hallucinations, apathy, depression, irritability, aberrant motor behavior, sleep disturbance, eating disorders) occur later or worsen in the MCI group, which may indicate their subsequent conversion to $\mathrm{AD}$. Indeed, these specific BPS are also found in the early stages of $\mathrm{AD}$. For example, apathy as the most frequent BPS in early $\mathrm{AD}$, followed by depression and irritability, are also reported by other investigators (Mega et al., 1996; Lyketsos et al., 2002; Holtzer et al., 2003; Pocnet et al., 2013) and found to be correlated with atrophy, hypoperfusion, or hypometabolism in the frontal and anterior cingulate cortices (Craig et al., 1996; Zubenko, 2000; Apostolova et al., 2007). Therefore, some BPS may have a neurobiological basis related to the neurodegenerative process. One might think that the changes in specific BPS occur jointly with some cognitive changes, even if the cognitive impairment can be very subtle at first in MCI individuals. 
Contrary to what we expected in the beginning, our findings show no link between depression and apathy and cognitive decline, although both depression and apathy are symptoms that tend to worsen over time. However, other specific changes in BPS were associated with a rapid deterioration of the global cognitive level in MCI patients. In particular, an increase of euphoria, eating disorders, and aberrant motor behavior, as well as worsened sleep quality, predicted decline in cognitive functioning. Copeland et al. (2003) explain that the excitement that accompanies some of these symptoms may affect cognitive abilities such as attention or working memory. In our study, the global cognitive functioning was evaluated using MMSE, which does not seem sensitive enough to detect progression of cognitive impairment over time among very mildly impaired individuals. In addition, the period of two years to follow-up was not enough to accurately estimate the rate of conversion of individuals with MCI to AD-type dementia. It is possible that the memory problems will progress within the next few years, but this does not necessarily mean that a diagnosis of $\mathrm{AD}$ will be made within the same follow-up period.

Unlike other studies (Ganguli et al., 2004), we did not find significant links between improvement of specific symptoms and a positive evolution of cognitive functioning, leading the people with MCI to revert to a normal level of cognitive functioning. Furthermore, no significant links between specific BPS and daily functioning were found, although minor changes of instrumental ADLs have been observed. This confirms previous findings on the preservation of everyday activities for some time in the MCI stage (Winblad et al., 2004).

Overall, our results are consistent with those of other studies that found that changes in specific neuropsychiatric symptoms would predict the progression of cognitive decline in individuals with MCI (Bozoki et al., 2001; Petersen, 2004; Monastero et al., 2006; Petersen, 2007; Monastero et al., 2009).

\section{Strengths and limitations of the study}

The sample of amnestic MCI patients was small and this may have led to an underestimation of the association of changes in specific BPS on cognitive decline. Hence, replication of this study in a larger sample is mandatory. Life events or socioeconomic status should be taken into account as these could better explain BPS fluctuation. Although the analyses were adjusted for significant age or level of education differences, findings ought to be replicated with more similar groups. Furthermore, detailed information on psychiatric antecedents was unavailable. The strength of our study ensues mainly from the use of well-validated instruments allowing to compare the two well-characterized clinical groups with the healthy control participants.

\section{Perspectives and practical implications}

Our findings hold important implications for the field of ageing psychology since people with MCI must cope differently in this transitional period compared to healthy people without cognitive disorders. Clinicians need to consider that the spectrum of symptom changes is not linear and that some symptoms may disappear or be replaced with others, which may influence the cognitive abilities of patients with MCI. Understanding the relationship between neuropsychiatric pattern changes and cognitive and functional decline may enable a better prediction of the evolution of this disease.

\section{Conclusions}

Our findings confirm a higher variability of BPS over time in amnestic single and multi-domain MCI than in AD. Moreover, our results provide evidence of associations between cognitive and noncognitive factors in the MCI group, suggesting that changes in some BPS could favor cognitive decline. However, longitudinal study designs, including both biological and psychosocial parameters, will shed light on the dynamics of developmental processes that may explain the observed differences. Refining our understanding of these processes is necessary to respond effectively to the demands of an aging society and the increasing number of patients with dementia.

\section{Conflict of interest}

None.

\section{Description of authors' roles}

Cornelia Pocnet: collected the $\mathrm{AD}$ and healthy data, and participated in the MCI data collection, carried out some statistical analysis, wrote the manuscript;

Jean-Philippe Antonietti: responsible for the statistical design and creating figures;

Alessia Donati: supervised and collected MCI data;

Julius Popp: participated in the MCI data collection and provided feedback for writing the paper; 
Jérôme Rossier: participated in the implementation of the project and provided feedback for writing the paper;

Armin von Gunten: designed the MCI study, carried it out, and provided feedback for writing the paper.

\section{Acknowledgments}

This research was financed by a Swiss National Science Foundation research grant FNS 3200B0122263 and a grant from the Swiss Alzheimer Association to Armin von Gunten and Jérôme Rossier.

\section{References}

Akaike, H. (1974). A new look at the statistical model identification. IEEE Transactions on Automatic Control, 19, 716-723. doi:10.1109/TAC.1974.1100705.

Anchisi, D., Borroni, B. and Franceschi, M. (2005). Heterogeneity of brain glucose metabolism in mild cognitive impairment and clinical progression to Alzheimer disease. Archives of Neurology, 62, 1728-1733.

Apostolova, L. G. et al. (2007). Structural correlates of apathy in Alzheimer's disease. Dementia and Geriatric Cognitive Disorders, 24, 91-97. doi:10.1059/000103914.

Barnes, D. E., Alexopoulos, G. S., Lopez, O. L., Williamson, J. D. and Yaffe, K. (2006). Depressive symptoms, vascular disease, and mild cognitive impairment. Archives General Psychiatry, 63, 273-280. doi:10.1001/archpsyc.63.3.273.

Bennett, D. A. et al. (2002). Natural history of mild cognitive impairment in older persons. Neurology, 59, 198-205. doi: 10.1212/WNL.59.2.198.

Bozoki, A., Giordani, B., Heidebrink, J. L., Berent, S. and Foster, N. L. (2001). Mild cognitive impairments predict dementia in nondemented elderly patients with memory loss. Archives of Neurology, 58, 411-416. doi:10.1001/archneur.58.3.411.

Buschke, H. (1984). Cued recall in amnesia. Fournal of Clinical and Experimental Neuropsychology, 6, 433-440.

Busse, A., Bischkopf, J., Riedel-Heller, S. G. and Angermeyer, M. C. (2003). Mild cognitive impairment: prevalence and incidence according to different diagnostic criteria: results of the Leipzig longitudinal study of the aged. Brain fournal of Psychiatry, 182, 449-454.

Copeland, M. P., Daly, E., Hines, V., Carol, M. M., Gunther, J. and Albert, M. (2003). Psychiatric symptomatology and prodromal Alzheimer's disease. Alzheimer Disease and Association Disorders, 17, 1-8.

Craig, A. H. et al. (1996). Cerebral blood flow correlates of apathy in Alzheimer disease. Archives of Neurology, 53, 1116-1120.

Cummings, J. L. (1997). The neuropsychiatric inventory: assessing of psychopathology in dementia patients. Neurology, 48, 10-16. doi:10.1212/WNL.48.5_Suppl_6.10S.
Cummings, J. L. and McPherson, S. (2001). Neuropsychiatric assessment of Alzheimer's disease and related dementias. Aging, 13, 240-246.

Folstein, M. F., Folstein, S. E. and McHugh, P. R. (1975). Mini-mental state: a practical method for grading the cognitive state of patients for clinicians. Fournal of Psychiatry Research, 12, 189-198. doi:10.1016/0022-3956 (75) 90026-6.

Ganguli, M., Dodge, H. H., Shen, C. and DeKosky, T. (2004). Mild cognitive impairment, amnestic type: an epidemiologic study. Neurology, 63, 115-121. doi: 10.1212/01.WNL.0000132523.27540.81

Geda, Y. E., Knopman, D. S. and Mrazeck, D. A. (2006). Depression, apoliproteine E genotype, and the incidence of mild cognitive impairment: a prospective cohort study. Archives of Neurology, 63, 435-440. doi:10.1001/archneur.63.3.435

Godefroy, O. et al. (2010). Dysexecutive syndrome: diagnostic criteria and validation study. American Neurological Association, 68, 855-864. doi. 10.1002/ana. 22117

Holtzer, R., Tang, M. X., Devanand, D. P., Albert, S. M. and Wegesin, D. J. (2003). Psychopathological features in Alzheimer's disease: course and relationship with cognitive status. Fournal of American Geriatrics Society, 51, 953-960. doi:10.1046/j.1365-2389.2003.51308.x

Jorm, A. F. and Jacomb, P. A. (1989). The informant questionnaire on cognitive decline in the elderly (IQCODE): socio-demographic correlates, reliability, validity, and some norms. Psychological Medecine, 19, 1015-1022. doi:10.1017/S0033291700005742.

Katz, I. (1998). Diagnosis and treatment of depression in patients with Alzheimer's disease and other dementias. Fournal of Clinical Psychiatry, 59, 38-44.

Lam, L. C., Leung, T., Lui, V. W., Leung, V. P. and Chiu, H. F. (2006). Association between cognitive function, behavioural syndromes and two-year clinical outcome in Chinese subjects with late-onset Alzheimer's disease. International Psychogeriatric, 18, 517-526. doi: $10.1017 /$ S1041610205002930.

Lawton, M. P. and Brody, E. M. (1969). Assessment of older people: self-maintaining and instrumental activities of daily living. Gerontologist, 9, 179-186. doi:10.1093/geront/9.3_Part_1.179.

Lyketsos, C. G., Lopez, O., Jones, B., Fitzpatrick, A. L., Breitner, J. and DeKosky, S. (2002). Prevalence of neuropsychiatric symptoms in dementia and mild cognitive impairment. Fournal of American Medical Association, 288, 1475-1483. doi:10.1001/jama.288.12.1475.

McKhann, G., Drachman, D., Folstein, M., Katzman, R., Price, D. and Stadlan, E. M. (1984). Clinical diagnosis of Alzheimer's disease: report of the NINCDS-ADRDA work group under the auspices of the department of health and human services task forces on Alzheimer's disease. Neurology, 34, 939944.

Mega, M. S., Cummings, J. L., Fiorello, T. and Gornbein, J. (1996). The spectrum of behavioural changes in Alzheimer's disease. Neurology, 46, 130-135. doi:10.1212/WNL.46.1.130

Modrego, P. J. and Ferrandez, J. (2004). Depression in patients with mild cognitive impairment increases the risk 
of developing dementia of Alzheimer type: a prospective cohort study. Archive Neurological, 61, 1290-1293. doi:10.1001/archneur.61.8.1290.

Monastero, R., Mangialasche, F., Camarda, C., Ercolani, S. and Camarda, R. (2009). A systematic review of neuropsychiatric symptoms in mild cognitive impairment, Fournal of Alzheimer's Disease, 18, 11-30. doi: 10.3233/JAD-2009-1120.

Monastero, R., Mariani, E., Camarda, C., Ingegni, T., Averna, M. R. and Senin, U. (2006). Association between apolipoprotein E4 allele and apathy in probable Alzheimer's disease. Acta Psychiatrica Scandinavica, 113, 59-63. doi:10.1111/j.1600-0447.2005.00597.x

Monastero, R., Palmer, K., Qiu, C., Winblad, B. and Fratiglioni, L. (2007). Heterogeneity in risk factors for cognitive impairment, no dementia: population-based longitudinal study from the Kungsholmen project. American fournal of Geriatric Psychiatry, 15, 60-69.

Morris, J. C. (1993). The clinical dementia rating (CDR): current version and scoring rules. Neurology, 43, 2412-2414.

Morris, J. C., Storandt, M. and Miller, J. P. (2001). Mild cognitive impairment represents early-stage Alzheimer disease. Archive Neurology, 58, 397-405.

doi:10.1001/archneur.58.3.397.

Nguyen, H. T. and Zonderman, A. B. (2006). Relationship between age and aspects of depression: consistency and reliability across two longitudinal studies. Psychological Aging, 21, 119-126. doi:10.1037/ 0882-7974.21.1.119.

Palmer, K., Berger, A. K., Monastero, R., Winblad, B., Bäckman, L. and Fratiglioni, L. (2007). Predictors of progression from mild cognitive impairment to Alzheimer disease. Neurology, 68, 1596-1602. doi:10.1212/01.wnl.0000260968.92345.3f.

Petersen, R. C. (2004). Mild cognitive impairment as a diagnostic entity. Fournal of Internal Medicine, 256, 183-194. doi:10.1111/j.1365-2796.2004.01388.x

Petersen, R. C. (2007). The current status of mild cognitive impairment-what do we tell our patients? Natura Clinical Practice Neurology, 3, 60-61. doi:10.1038/ ncpneuro0402.
Pocnet, C., Rossier, J., Antonietti, J.-Ph. and von Gunten, A. (2013). Personality traits and behavioral and psychological symptoms in patients at an early stage of Alzheimer's disease. International fournal of Geriatric Psychiatry, 28, 276-283. doi:10.1002/gps.3822.

R Development Core Team. (2009). R: A Language and Environment for Statistical Computing. Vienna, Austria: R Foundation for Statistical Computing.

Ritchie, K. et al. (2010). Retrospective identification and characterization of mild cognitive impairment from a prospective population cohort. American foumal of Geriatric Psychiatry, 18, 692-700. doi: 10.1097/JGP.0b013e3181df4897

Rozzini, L., Chilovi, B. V., Trabucchi, M. and Padovani, A. (2005). Depression is unrelated to conversion to dementia in patients with mild cognitive impairment. Archives Neurology, 62, 505. doi:10.1001/archneur.62.3.505-a

Teng, E., Lu, P. H. and Cummings, J. L. (2007). Neuropsychiatric symptoms are associated with progression from mild cognitive impairment to Alzheimer's disease. Dementia and Geriatric Cognitive Disorders, 24, 253-259. doi: 10.1159/000107100.

Winblad, B., Palmer, K. and Kivipelto, M. (2004). Mild cognitive impairment-beyond controversies, towards a consensus: report of the international working group on mild cognitive impairment. Fournal International Medicine, 256, 240-246. doi:10.1111/j.1365-2796.2004.01380.x

Word Health Organization. (1993). The ICD-10 Classification of Mental and Behavioral Disorders: Diagnostic Criteria for Research, Geneva: Word Health Organization.

Yaffe, K., Blackwell, T., Gore, R., Sands, L., Reus, V. and Browner, W. S. (1999). Depressive symptoms and cognitive decline in nondemented elderly women. Archives of General Psychiatry, 56, 425-430. doi:10.1001/archpsyc.56.5.425.

Zigmond, A. S. and Snaith, R. P. (1983). The hospital anxiety and depression scale. Acta Psychiatrica Scandinavica, 67, 361-370. doi:10.1111/j.1600-0447.1983.tb09716.x

Zubenko, G. S. (2000). Neurobiology of major depression in Alzheimer's disease. International Psychogeriatrics, 12, 217-230. 